\title{
Prática eSPORTIVA E USO de SUbSTÂnCIAS ENTRE ESTUdANTES do ENSINo MÉDIO: DIFERENTES PERSPECTIVAS DESSA RELAÇÃO
}

\author{
André Bedendo \\ André Luiz Monezi Andrade ${ }^{2}$ \\ Ana Regina Noto ${ }^{3}$
}

O presente estudo teve por objetivo descrever a relação entre a prática de esportes e o consumo de substâncias entre estudantes do ensino médio. Realizou-se uma busca, sem restrição de data, nas principais bases de dados sobre saúde (Cochrane, LILACS, PsycINFO, PubMed, SciELO e Scopus). A análise evidenciou que a prática esportiva pode se apresentar tanto como fator associado à proteção quanto ao risco de uso, dependendo de uma série de variáveis. Entre essas se destacam o gênero, as modalidades esportivas, o ambiente sociocultural e motivação tanto para a prática esportiva quanto para o consumo da substância. O planejamento de ações preventivas que envolvam a prática de esportes devem considerar os diferentes fatores envolvidos, a fim de promover a prevenção do consumo de drogas entre os adolescentes.

Descritores: Esportes; Transtornos Relacionados ao Uso de Substâncias/prevenção \& controle; Adolescente; Estudantes.

\footnotetext{
${ }^{1}$ Doutorando, Universidade Federal de São Paulo, São Paulo, SP, Brasil. Bolsista da Fundação de Amparo à Pesquisa do Estado de São Paulo (FAPESP), Brasil.

2 Doutorando, Universidade Federal de São Paulo, São Paulo, SP, Brasil. Bolsista do Conselho Nacional de Desenvolvimento Científico e Tecnológico (CNPq), Brasil. Professor, Centro Regional de Referência, São Paulo, SP, Brasil. Professor, Universidade Anhembi Morumbi, São Paulo, SP, Brasil.

${ }^{3}$ PhD, Professor Adjunto, Departamento de Psicobiologia, Universidade Federal de São Paulo, São Paulo, SP, Brasil
}

\author{
Correspondencia: \\ André Bedendo \\ Universidade Federal de São Paulo. Departamento de Psicobiologia \\ Ed. Ciências Médicas, $1^{\circ}$ andar \\ Rua Botucatu, 862 \\ Vila Mariana \\ CEP: 04023-062, São Paulo, SP, Brasi \\ E-mail: andrebedendo@gmail.com
}




\section{SPORTS AND SUBSTANCE USE IN HIGH SCHOOL STUDENTS: DIFFERENT PERSPECTIVES OF THIS RELATIONSHIP}

The aim of this study was to describe the relationship between playing sports and substance use in high school, students. A search was conducted, without restrictions by date, in the main health databases saúde (Cochrane, LILACS, PsycINFO, PubMed, SciELO and Scopus). Analysis showed that playing sports can be both a risk and a protection factor concerning substance use, depending on a series of variables. Among these the following stand out: gender, type of sport, socio-cultural environment and motivation, both to play sport and to take the substance. Planning preventative actions that involve sport should consider the various factors involved, so as to encourage prevention of drug consumption among adolescents.

Descriptors: Sports; Substance-Related Disorders/prevention \& control; Adolescent; Students.

\section{Practica deportiva y el uso de sustancias entre estudiantes de SECUNDARIA: DIFERENTES PERSPECTIVAS DE ESTA RELACIÓN}

El presente estudio tuvo por objetivo describir la relación entre practica deportiva y el consumo de sustancias entre los estudiantes de secundaria. Se realizo una búsqueda sin restricción de fecha, en la principales bases de datos sobre salud. (Cochrane, LILACS, PsycINFO, PubMed, SciELO e Scopus). El análisis puso en evidencia que la practica deportiva se puede presentar tanto como un factor asociado a la protección, como también un factor de riesgo para el consumo, dependiendo de una series de variables. Entre estas se destacan el genero, las modalidades deportivas, el ambiente sociocultural y la motivación tanto para la practica deportiva, cuanto para el consumo de las sustancias. La planeación de acciones preventivas que incluyan la practica de deportes deben considerar los diferentes factores implicados, con el fin de promover la prevención de el consumo de drogas entre los adolescentes.

Descriptores: Deportes; Trastornos Relacionados con Sustancias/Prevención \& Control; Adolescente; Estudiantes.

\section{Introdução}

A adolescência é um período permeado por importantes mudanças neuroanatômicas e funcionais e que estão associadas a comportamentos de maior ansiedade, impulsividade e insegurança se comparados a adultos ${ }^{(1-2)}$. Por outro lado, também há uma série de novas relações sociais que são particularmente especiais na vida do indivíduo, sejam elas na relação com os pais ou grupos de amigos. Nesse caso, se algum desses grupos consome drogas, pode haver pressão para o consumo do indivíduo, e uma vez em contato com as drogas há também maior exposição aos diversos riscos associados ao consumo ${ }^{(3)}$. 
O abuso de substâncias por adolescentes está associado a diversos problemas sociais e de saúde, como acidentes automobilísticos, violência, problemas acadêmicos e prejuízos de memória $^{(4-7)}$. Dessa forma, há substancial esforço na prevenção do uso de substâncias entre estudantes dessa faixa etária ${ }^{(8)}$. Entre as possíveis estratégias de prevenção, a prática de esportes tem sido sugerida como uma opção ao uso de drogas entre adolescentes $^{(9)}$.

Estudos sugerem que, do ponto de vista neurobiológico, a prática de esportes pode ser alternativa interessante para evitar ou diminuir o consumo de drogas ${ }^{(10)}$. Entretanto, o consumo de substâncias não se resume a esse único fator, sendo também importante o contexto em que o indivíduo se insere. Consequentemente, ainda existem lacunas na literatura sobre algumas especificidades da relação entre prática de esporte e uso de drogas ${ }^{(11)}$, principalmente em relação à atuação da prática esportiva, enquanto fator associado ao risco ou proteção para o uso e abuso de drogas.

Dessa forma, o presente trabalho teve por objetivo descrever a relação entre a atividade esportiva e o uso de drogas, explorando variáveis relacionadas a partir da literatura disponível sobre a relação entre a prática de esportes e o uso de substâncias por adolescentes estudantes do ensino médio.

\section{Metodologia}

O presente trabalho configura-se como uma revisão narrativa da literatura. Foram pesquisadas as seguintes bases de dados Cochrane, LILACS, PsycINFO, PubMed, SciELO e Scopus, sem restrição de data, utilizando-se o cruzamento das palavras-chave: "sports" e "drug abuse", "drug misuse", "substance abuse", "addiction". A busca ocorreu em janeiro de 2011.

Os critérios de inclusão utilizados foram: estudo da relação entre prática esportiva e uso de drogas psicotrópicas (passíveis de autoaplicação e com potencial para causar dependência, artigos originais publicados em português, inglês, ou espanhol e estudantes do ensino médio. O seguinte algoritmo de busca foi utilizado: (tw:(sports)) AND (tw:(drug abuse)) AND (tw:(drug misuse)) AND (tw:(substance abuse)) AND (tw:(addiction)) AND (limit: ("secondary students") AND la:(“en" OR “es" OR “pt”) AND type: ("article”)).

Há autores que estudaram exclusivamente drogas que não são descritas com potencial para causar dependência, por exemplo, hormônio do crescimento e eritroproteína, ou que envolviam comorbidades, essas não foram consideradas nesta revisão.

\section{Resultados}

As buscas não envolveram restrição de data e retornaram 6.843 artigos, dos quais as referências duplicadas foram excluídas $(n=3.646)$. Aplicaram-se então os critérios de inclusão, sendo 1.774 referências excluídas pelo título, 38 por não serem artigos publicados em periódicos científicos e 1.201 através do resumo. Foram encontradas 184 referências potencialmente relevantes, das quais os textos completos foram avaliados. Quatro outros trabalhos, publicados posteriormente a essa data - referências $22,32,41$ e 49 -, foram considerados importantes pelos autores e foram incluídos nesta revisão. Para esta revisão, foram utilizados 28 artigos recolhidos (Figura 1). As informações sobre autores, ano de publicação, amostra, idade, país de origem, tipos de esportes e drogas estudadas são apresentadas na Figura 2. 


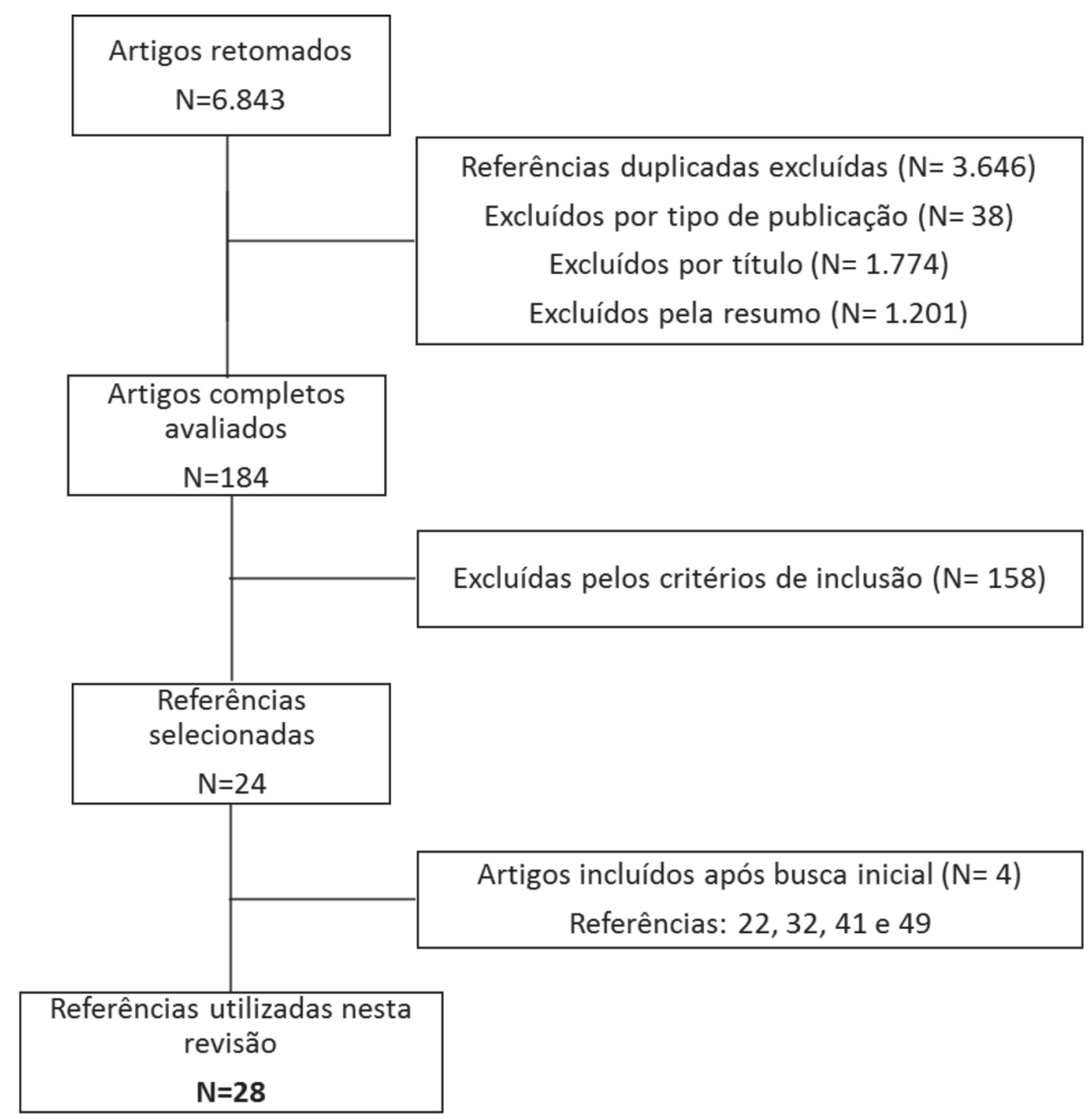

Figura 1 - Fluxograma de exclusão de artigos 


\begin{tabular}{|c|c|c|c|c|c|c|c|c|c|c|c|c|c|c|c|c|c|}
\hline 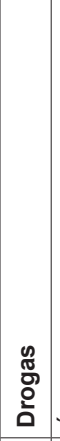 & 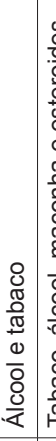 & 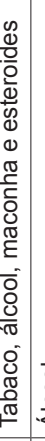 & & 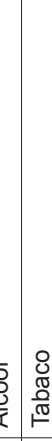 & & 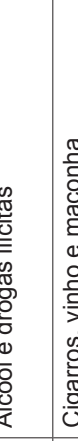 & 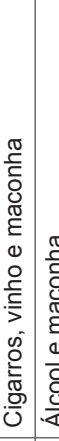 & 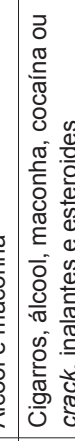 & 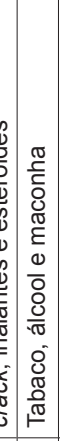 & 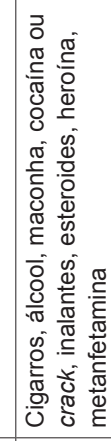 & & $\begin{array}{l} \\
\bar{o} \\
\frac{\mathrm{o}}{4}\end{array}$ & 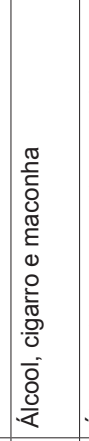 & 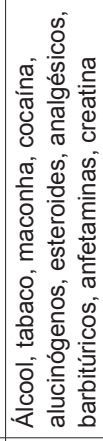 & 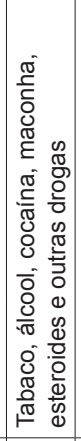 & 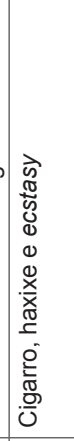 & 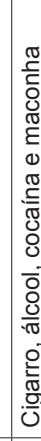 \\
\hline 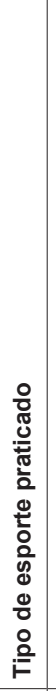 & 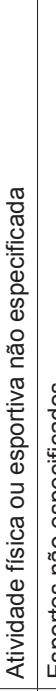 & 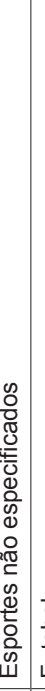 & 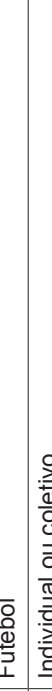 & 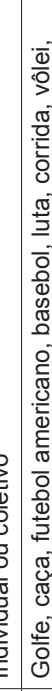 & 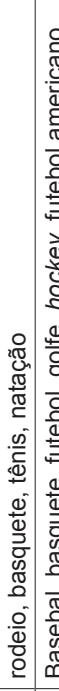 & 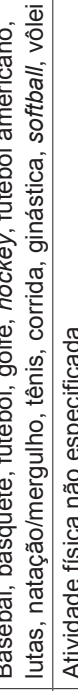 & 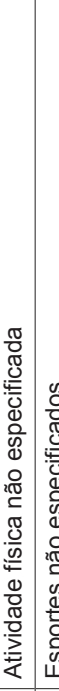 & 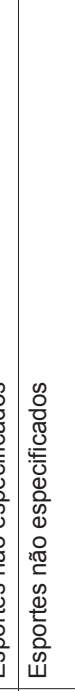 & 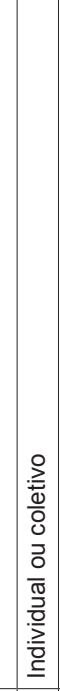 & 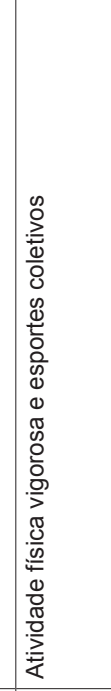 & 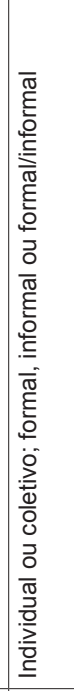 & 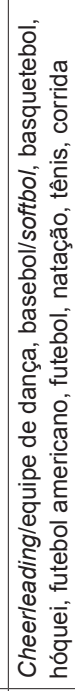 & 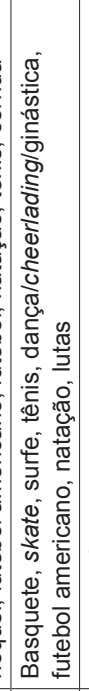 & 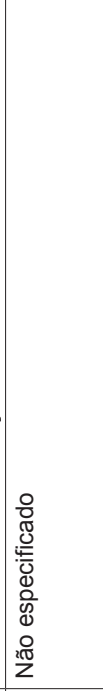 & 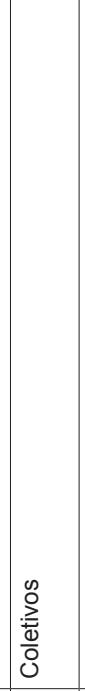 & 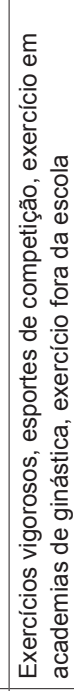 & 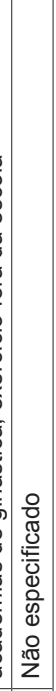 \\
\hline 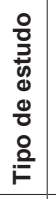 & 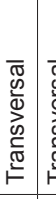 & 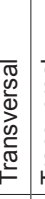 & 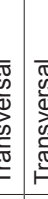 & 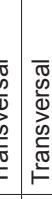 & & 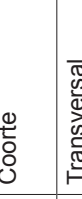 & 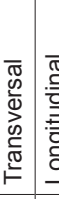 & 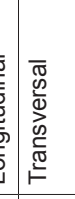 & 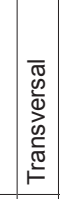 & 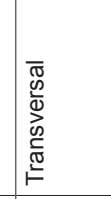 & 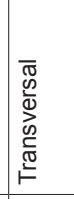 & 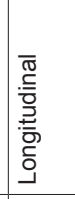 & 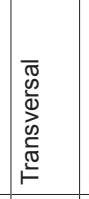 & 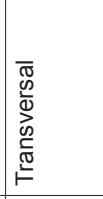 & 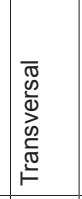 & 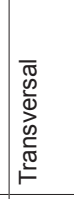 & 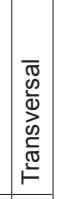 \\
\hline 这 & 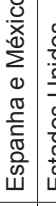 & 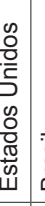 & 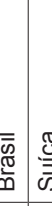 & 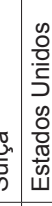 & \begin{tabular}{|l}
$y$ \\
$\frac{0}{0}$ \\
$\frac{0}{0}$ \\
5 \\
0 \\
0 \\
$\frac{0}{0}$ \\
$\frac{\pi}{5}$ \\
$\frac{5}{4}$
\end{tabular} & 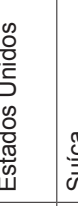 & 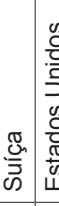 & 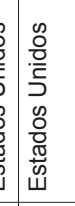 & 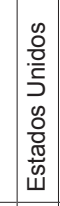 & 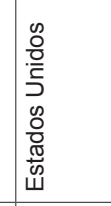 & 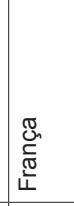 & 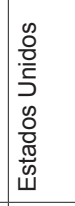 & 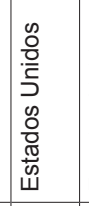 & 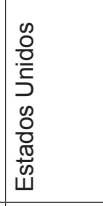 & 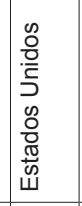 & 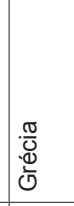 & 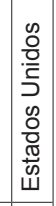 \\
\hline 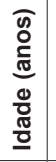 & $\begin{array}{l}0 \\
0 \\
0 \\
\\
\end{array}$ & $\begin{array}{l}\infty \\
\frac{\infty}{\sigma} \\
\stackrel{\sigma}{\leftarrow}\end{array}$ & 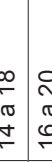 & 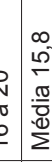 & & 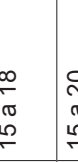 & 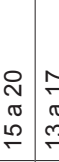 & $\begin{array}{l}\frac{5}{0} \\
\frac{\pi}{7} \\
y\end{array}$ & 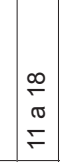 & $\begin{array}{l}\infty \\
\infty \\
\sigma \\
\sigma \\
\sim\end{array}$ & 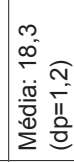 & $\underset{\substack{\infty \\
\underset{N}{\infty}}}{\stackrel{\infty}{\sim}}$ & 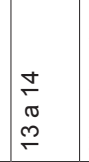 & 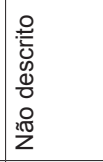 & 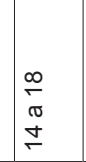 & $\begin{array}{l}\mathscr{O} \\
\sigma \\
\Gamma\end{array}$ & 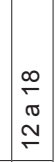 \\
\hline 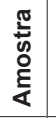 & 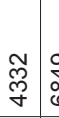 & 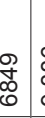 & 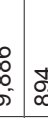 & t. & & 啇 & 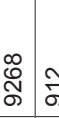 & {$\left[\begin{array}{l}\infty \\
0 \\
0 \\
m\end{array}\right.$} & 弚 & 扂 & $\stackrel{0}{\infty}$ & 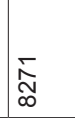 & |', & 占 & $\begin{array}{l}\mathcal{N} \\
\underset{N}{N}\end{array}$ & 㽞 & \\
\hline$\stackrel{\circ}{\frac{一}{4}}$ & $\stackrel{N}{\tilde{N}}$ & $\begin{array}{l}\infty \\
\stackrel{\infty}{\circ} \\
\end{array}$ & 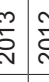 & $\begin{array}{l}\vec{v} \\
v \\
\end{array}$ & & \& & \begin{tabular}{l|l}
$\stackrel{8}{\circ}$ \\
\end{tabular} & s. & $\begin{array}{l}\infty \\
\infty \\
\stackrel{2}{\longrightarrow} \\
\stackrel{\rho}{\longrightarrow} \\
\end{array}$ & ֻे & 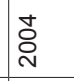 & $\stackrel{\circ}{\stackrel{2}{2}}$ & 苂 & চ্ণু & 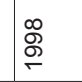 & ఫે & $\stackrel{\leftrightarrow}{\stackrel{\leftrightarrow}{\circ}}$ \\
\hline 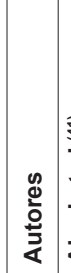 & 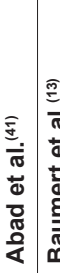 & 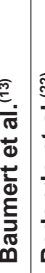 & 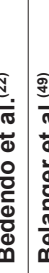 & 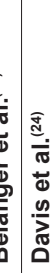 & 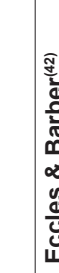 & 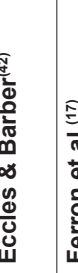 & 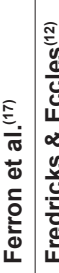 & 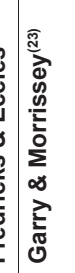 & 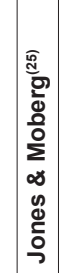 & 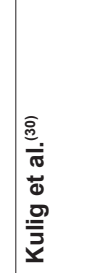 & 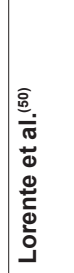 & 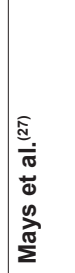 & 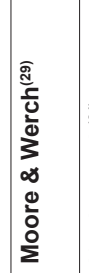 & 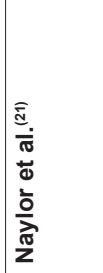 & 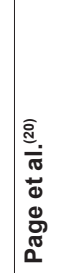 & 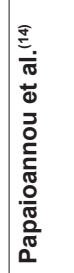 & 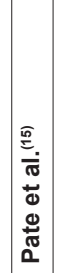 \\
\hline
\end{tabular}




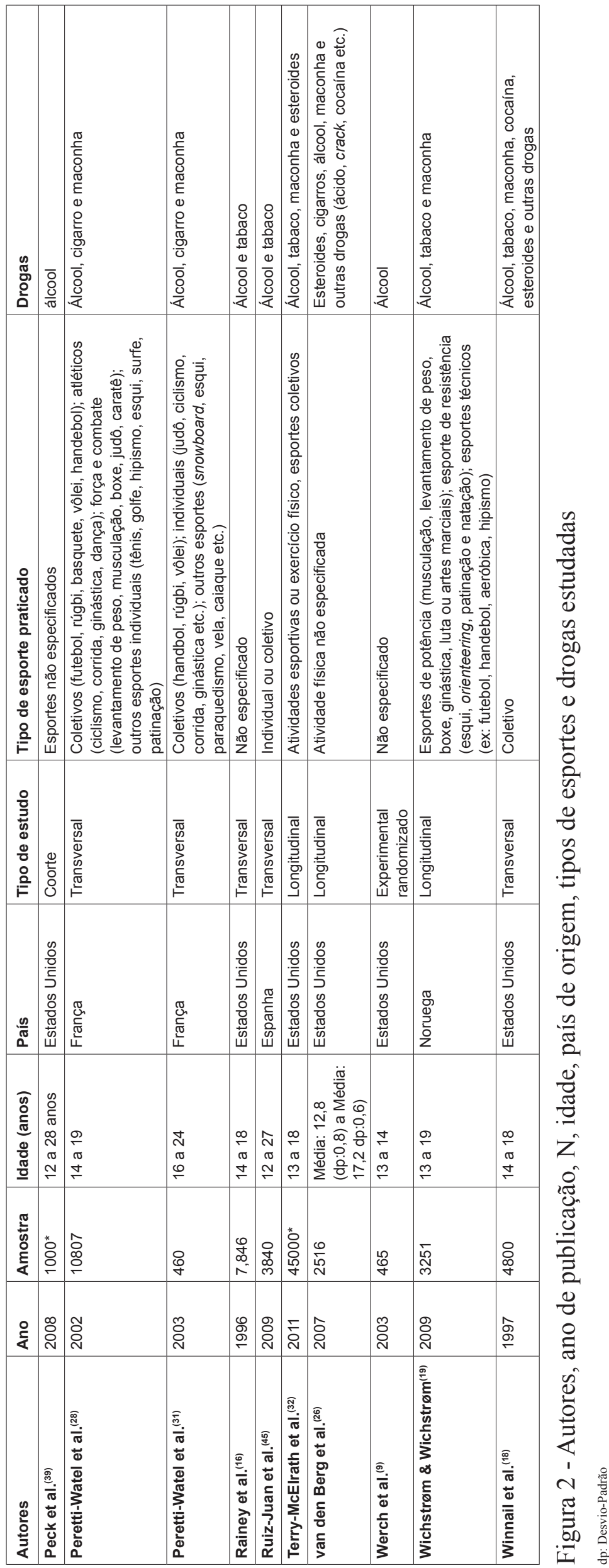


Tipos de drogas

Vários estudos buscam elucidar a relação entre a prática de esporte e uso de substâncias entre adolescentes. Em outros estudos observou-se que a prática de atividades esportivas está relacionada ao menor consumo de álcool ${ }^{(12)}$, tabaco ${ }^{(13-18)}$, maconha $^{(13,19-20)}$ e cocaína ${ }^{(20-21)}$. Por sua vez, outros estudos referem maiores prevalências entre praticantes de esportes, comparados a não praticantes. Entre as substâncias estão o uso de álcool ${ }^{(16,18,22-23)}$, tabaco $^{(24-25)}$ e esteroides anabolizantes ${ }^{(26)}$.

Resultados tão contrastantes podem estar relacionados a uma série de fatores metodológicos, dentre eles diferentes populações ou amostras, variações entre os instrumentos de pesquisa ou entre as variáveis estudadas, até mesmo diferentes objetivos de cada estudo. Além disso, a relação entre a prática de esportes e uso de drogas parece não ser tão direta uma vez que existem diversas variáveis terciárias envolvidas. Entre as principais variáveis observadas nesta revisão, destacaram-se a influência do gênero, tipos de esportes (coletivos ou individuais), motivações (para a prática e para o consumo da substância) e o ambiente social e cultural no qual o praticante está inserido.

\section{Diferenças entre gênero}

Alguns autores sugerem que a relação entre esportes e o uso problemático de álcool independe se entre homens e mulheres ${ }^{(20,27)}$. Por outro lado, a maioria dos trabalhos aponta diferenças entre gênero. Em um estudo longitudinal, observou-se maior consumo de álcool entre mulheres que haviam praticado esportes durante o ensino médio, entretanto, o mesmo não foi observado entre os homens ${ }^{(12)}$. Em outro trabalho, avaliando estudantes franceses do ensino médio, observaram-se diferenças entre homens e mulheres tanto em relação aos padrões de consumo de álcool, tabaco e maconha ${ }^{(28)}$ quanto em relação aos esportes praticados. Homens praticavam mais esportes de força e combate (lutas, levantamento de peso etc.), enquanto mulheres praticavam mais esportes atléticos (ginástica, dança etc.). Dessa forma, os autores destacaram que as diferenças de gênero, relacionadas ao consumo de drogas, parecia estarem parcialmente relacionadas aos tipos de esportes praticados. Outro estudo apontou que mulheres que praticavam esportes patrocinados pela escola tinham menor risco para consumo de álcool, enquanto homens, na mesma condição, têm maior risco para o uso de substâncias. Entretanto, essas relações tiveram forte influência do tipo de esporte praticado ${ }^{(29)}$.

Apesar de não haver impedimentos, muitos esportes tendem a ser considerados mais como atividades masculinas do que femininas, e vice-versa. Por exemplo, o futebol no Brasil é histórico e culturalmente um esporte predominantemente masculino, enquanto dança e ginástica tendem a ser predominantemente femininos. Para homens, muitas vezes, o esporte se configura como uma forma de evidenciar os valores masculinos, por exemplo, através da demonstração de força ou agressividade ${ }^{(30)}$. Da mesma maneira, o consumo exagerado de álcool por álcool também já foi descrito como maneira de expressar esses valores masculinos.

\section{Esportes coletivos e individuais}

Alguns trabalhos apontam que a relação entre a prática esportiva e o uso de substâncias também varia dependendo do tipo de esporte praticado $^{(31)}$. Esses estudos demonstraram que alguns esportes como natação, mergulho, snowboard e paraquedismo se associam mais com o consumo de determinadas substâncias, como álcool e maconha, quando comparados com outras atividades esportivas.

Um estudo de seguimento de 13 anos com estudantes de ensino médio mostrou que praticantes de esportes coletivos, quando comparados aos de esportes individuais, apresentaram maior aumento no número de intoxicações por álcool ao longo dos anos ${ }^{(19)}$. A partir de uma amostra representativa de estudantes norte-americanos, por meio de metodologia transversal (entre 1991 e 2009), evidenciou que altos níveis de prática de esportes coletivos estavam associados a maior uso de tabaco não fumado (por exemplo, mascado ou inalado) e baixos níveis de uso (mensal) de cigarros e maconha. Maior consumo de álcool e esteroides anabolizantes no último mês foi observado somente entre estudantes dos últimos anos do ensino médio ${ }^{(32)}$. No entanto, esses autores não analisaram dados de esportes individuais. Além disso, os autores de ambos os estudos citados não descrevem detalhadamente quais as atividades esportivas compreendidas sob o escopo de esportes coletivos, dificultando a interpretação dos resultados.

Adolescentes homens, praticantes de esportes coletivos, consumiam menos drogas como cocaína, heroína, metanfetamina, crack que seus pares de esportes não coletivos, no entanto, não 
foram encontradas associações significativas com o uso de álcool ${ }^{(30)}$. Os autores destacaram que se existe essa relação entre o comportamento de risco e esportes coletivos que parece ocorrer diferentemente entre homens e mulheres. Além disso, os autores destacaram o medo de prejudicar o time, uma vez que o uso de drogas tende a ser prejudicial à performance esportiva, pode agir no sentido de evitar o consumo.

A prática esportiva sempre implica algum nível de socialização, seja coletivo ou não. A principal diferença nesse caso reside no fato de como o praticante se relaciona com os demais participantes, uma vez que, no esporte coletivo, existem tanto oponentes quanto parceiros de time. Assim, as possibilidades de contato social e interação de praticantes de esportes coletivos tendem a ser diferentes daquelas de esportistas individuais, proporcionando ofertas ambientais também diferenciadas. Em esportes coletivos, a interação entre os atletas do time em contextos que não envolvam necessariamente o esporte praticado (por exemplo, confraternizações ou festas) pode ser mais frequente. Alguns trabalhos indicam que atletas parece que se engajam mais no consumo de álcool com outros esportistas como forma de aumentar a coesão do time ${ }^{(33)}$. Além disso, talvez a prática de esportes coletivos, quando associada a viagens para longe de casa, como, por exemplo, para uma competição, possa facilitar que adolescentes experimentem bebidas alcóolicas.

A maior socialização não presume necessariamente maior uso de drogas, desde que o consumo não seja tido como normativo ou incentivado dentro do grupo. A teoria da autodeterminação postula que as necessidades de autonomia, competência e relacionamentos dos indivíduos se pautam em motivações extrínsecas e intrínsecas $^{(34)}$. Nesse sentido, o adolescente que queira se sentir integrado ao grupo, nesse caso com o time, tende a agir da mesma forma que seus pares, a fim de promover sua aceitação. Caso o uso de substâncias não seja bem visto pelo grupo, o adolescente tende a não consumir a droga ou diminuir seu uso. Dessa forma, para o planejamento de ações preventivas em ambiente esportivo, torna-se necessário avaliar o contexto sociocultural que envolve a equipe como um todo.

Aspectos sociais e culturais

Vários estudos destacaram a importância dos componentes sociais no consumo de substâncias entre adolescentes ${ }^{(35)}$. A teoria ecológica de Bronfenbrenner ${ }^{(36)}$ engloba vários níveis de influência social que podem atuar no indivíduo, desde o microssistema (as mudanças ocorrem através das relações interpessoais e num nível imediato) até no cronossistema (mudanças ocorridas ao longo do tempo como na estrutura familiar, status socioeconômico, local de residência). Essa teoria tem sido também utilizada como base teórica no estudo do uso de substâncias por adolescentes $^{(37-38)}$ e ajuda a compreender a complexidade que envolve a relação desse comportamento com a preferência e a prática esportiva.

A prática de esportes, como fator associado ao risco ou proteção ao uso de drogas, ainda é uma área inconsistente na literatura ${ }^{(39)}$. Nesse sentido, as diferenças entre os resultados podem em parte ser explicadas pelas diferenças culturais e sociais das amostras. No entanto, grande parte dos estudos falha ao descrever claramente o tipo de esporte e os padrões de uso de drogas, fatores fundamentais para a melhor compreensão dessa relação.

No Brasil, a prática do futebol é amplamente difundida, assim como o uso de bebidas alcoólicas, inclusive entre adolescentes de diferentes contextos socioeconômicos. A cerveja no Brasil é associada ao futebol, assim como nos EUA, essa relação ocorre com o futebol americano e beisebol ${ }^{(40)}$. Contudo, essa forte relação cultural entre a prática de esportes e o consumo de álcool também se faz presente em outros países e tende a afetar os adolescentes independentemente de serem praticantes de esportes ou sedentários, uma vez que são valores altamente prevalentes na cultura de certos países.

Em um estudo com estudantes de 12 a 16 anos da Espanha e México, os autores observaram que, entre os espanhóis, ser ativo em relação à prática esportiva foi evidenciado como fator de proteção ao uso de álcool e tabaco. No entanto, o mesmo não foi observado entre os estudantes mexicanos ${ }^{(41)}$. Outros dois trabalhos de um mesmo pesquisador apresentaram resultados divergentes. No primeiro, observou-se que praticantes de esporte relataram maior uso de álcool nos últimos seis meses, comparados aos não praticantes ${ }^{(42)}$. Em contrapartida, no segundo estudo observou-se que praticantes de esportes estavam menos associados ao menor uso de álcool no mesmo período ${ }^{(12)}$. Os próprios autores, por sua vez, destacaram que uma importante diferença em relação às duas populações estudadas não residia na prática esportiva, mas, sim, em 
diferenças históricas e demográficas das mesmas. O primeiro estudo compreendia uma amostra predominantemente de adolescentes brancos norte-americanos, enquanto que o segundo foi composto eminentemente de adolescentes afro-americanos e americanos de origem europeia. Dessa forma, os autores sugerem a possibilidade de que o uso de álcool seja culturalmente menos relevante entre afro-americanos do que entre os adolescentes brancos.

Atletas geralmente possuem mais oportunidades para participar de festas e o número de eventos sociais que frequentam estão associados ao uso de risco de álcool. Além disso, possuem mais amigos que consomem bebidas alcoólicas $^{(43)}$. Como forma de manter ou aumentar o status social, o atleta pode cessar ou diminuir o consumo de drogas, uma vez que o uso de substâncias não seja aceito como comportamento de um praticante de esportes. Por outro lado, é possível que exista aumento do uso de substâncias para se aumentar esse status social em um grupo que tenha o uso de drogas como normativo.

Adolescentes são mais susceptíveis que adultos a se engajarem em comportamentos de risco, como o uso de drogas, devido a uma série de mudanças que modulam funcional e estruturalmente seu cérebro, principalmente o sistema socioemocional, e quando se relacionam com seus pares ${ }^{(44)}$. Assim, é fundamental o estudo das relações sociais e fatores existentes na dinâmica do praticante de esporte que possam favorecer a diminuição ou aumento do consumo de substâncias. A partir desse conhecimento, projetos de intervenção e prevenção poderiam se beneficiar, favorecendo a criação de programas mais específicos e efetivos para a população de adolescentes.

\section{Motivações para prática esportiva e uso de drogas}

A motivação para a prática de uma modalidade esportiva, assim como para o uso de drogas, parece ser um importante parâmetro para compreensão sobre a relação entre uso de drogas e esportes. Em estudo realizado entre adolescentes de 12 a 16 anos, as motivações extrínsecas para a prática de esportes, como a aceitação social, não apresentaram relação com o uso de álcool ou tabaco. Todavia, a motivação intrínseca (sentir prazer e satisfação praticando ou competindo) foi mais associada a praticantes que não consumiam álcool e tabaco ${ }^{(41)}$. Em contrapartida, um estudo realizado com adolescentes espanhóis, inseridos no sistema público de ensino, não mostrou diferenças significativas quanto à motivação (intrínseca ou extrínseca) para a prática de esportes em relação ao consumo de álcool ou taba$\mathrm{co}^{(45)}$. Contudo, nesse estudo os autores analisaram uma amostra de estudantes com idade de 12 até 27 anos, inseridos em diversos níveis educacionais. Consequentemente, a amostra pode ter sido demasiadamente heterogênea, dificultando a comparação entre os trabalhos com população somente de adolescentes.

Existem diversos motivos que podem favorecer o consumo de drogas entre adolescentes. A maioria tende a relatar que consome álcool por motivos sociais, para se embriagar, relaxar, ou como estratégia de enfrentamento ${ }^{(46)}$. Determinados contextos da prática de esportes podem ser extremamente estressantes, especialmente aqueles em que há cobrança contínua por resultados. Dessa forma, o maior uso de substâncias por praticantes de esportes pode estar associado a uma estratégia de alívio de sintomas de ansiedade e estresse relacionado à rotina de treinos e competições ${ }^{(47-48)}$.

Por outro lado, o motivo para o consumo pode se pautar mais no contexto ambiental do que nas próprias motivações internas do indivíduo, como o uso de álcool por motivos sociais ou para se sentir bem media a relação entre o tipo de esportes e as várias medidas do uso de álcool. Além disso, praticantes de esportes coletivos podem beber para não serem desaprovados pelo grupo $^{(49)}$. Dessa forma, a fim de promover melhor inserção em determinado grupo, o adolescente tende a se moldar semelhantemente a seus pares para que se sentir mais acolhido.

\section{Considerações Finais}

O objetivo deste estudo foi apresentar algumas variáveis envolvidas com a prática esportiva e o uso de substâncias, a fim de fornecer subsídios para discussão sobre a temática. Entre a literatura revisada, as principais variáveis envolvidas foram o gênero, tipos de esportes (coletivos ou individuais), motivações (para a prática e para o consumo da substância) e o ambiente social e cultural.

A principal contribuição do presente trabalho foi evidenciar que a relação entre a prática de esportes e o uso de substâncias não é linear, mas dependente de uma série de variáveis referentes tanto ao contexto da prática quanto das características 
dos praticantes. Consequentemente, a prática de esportes per se não deve ser considerada fator associado à proteção ou ao risco para o uso de substâncias entre adolescentes.

Em quase todos os estudos levantados nesta revisão, a relação entre prática de esportes e uso de drogas foi estudada em populações dos Estados Unidos ou Europa, sendo poucos os estudos da América Latina. Isso reflete a lacuna de conhecimento sobre a relação entre esporte e drogas em países em desenvolvimento e sugere a necessidade de mais trabalhos nessas culturas.

Algumas das principais lacunas observadas nos estudos da área estão no fato de a maioria dos trabalhos não diferenciarem corretamente os tipos de esportes avaliados ${ }^{(11)}$. Além disso, já foi demonstrado previamente que o relacionamento entre o consumo de substâncias e a prática de esportes depende do tipo de esporte, sendo observadas particularidades, como relações específicas entre determinadas práticas e algumas substâncias ${ }^{(28)}$. Ainda, em relação às especificidades da atividade esportiva, a frequência da prática tende a variar de um estudo para outro, sendo esse um fator já descrito como diferenciador para o consumo de substâncias ${ }^{(50)}$.

Entre as substâncias estudadas, grande parte dos trabalhos focou o uso de tabaco e álcool. Quando abordadas as demais drogas, essas foram comumente classificadas dentro de uma única categoria englobando todas as drogas ilícitas, as quais, na verdade, são diversas em relação aos seus efeitos e contextos sociais de uso. Além disso, nem sempre os padrões de consumo são devidamente relatados, dificultando ainda mais a comparação dos trabalhos. Possivelmente isso seja devido à menor prevalência do uso das demais drogas. No entanto, são fundamentais futuros estudos sobre essas outras drogas, para melhor compreender a sua relação com práticas esportivas.

O conhecimento sobre os fatores associados ao abuso de substâncias é considerado um dos principais elementos no planejamento de ações preventivas $^{(8)}$. Esta revisão evidenciou que, apesar de ainda não se saber exatamente quais fatores apresentam associação ou não ao maior uso de drogas por adolescentes que praticam esportes, existem algumas variáveis que parecem modificar essa relação, como o gênero, tipo de esporte, motivações para a prática e para o consumo de drogas, o ambiente social e a cultura. Além dessas variáveis, outros fatores parecem estar relacionados ao uso de substâncias e à prática de esportes. Por exemplo, preocupações com sua própria saúde, sanções legais, sociais e autoimpostas podem favorecer o menor consumo por praticantes. Por outro lado, a aceitação do uso por pares, a busca por status e a identidade individual podem favorecer o maior uso de drogas. Outros fatores também podem atuar como uma espécie de gatilho para o início ou aumento do uso, como através da pressão social, a cultura de cada esporte e a necessidade de demonstrar masculinidade.

Diversas campanhas nacionais e internacionais sobre prevenção do uso de drogas veiculam o esporte como uma atividade protetiva ao consumo de substâncias. Entretanto, para que programas de prevenção ao abuso de drogas que incluam a prática esportiva sejam mais efetivos, devem ser consideradas as diferentes perspectivas que envolvem a questão. Os trabalhos que compõem esta revisão indicam que a associação entre a prática esportiva e o uso de substâncias por estudantes é um processo complexo, envolvendo uma série de fatores, o que pode torná-lo tanto fator de proteção quanto de risco. Nessa perspectiva, o esporte não deve ser considerado como um constructo único. As particularidades de cada prática esportiva e de seus praticantes devem ser consideradas para o planejamento de intervenções preventivas mais adequadas. Assim, práticas esportivas avaliadas como protetivas podem ser exploradas em campanhas preventivas. Em contrapartida, o reconhecimento de contextos, associados ao risco, também podem auxiliar no direcionamento de políticas públicas.

\section{Referências}

1. Chambers RA, Taylor JR, Potenza MN. Developmental neurocircuitry of motivation in adolescence: a critical period of addiction vulnerability. Am J Psychiatry. 2003;160:1041-52.

2. Toga AW, Thompson PM, Sowell ER. Mapping brain maturation. Trends Neurosci. 2006;29(3):801-9.

3. Marques ACPR, Cruz MS. O adolescente e o uso de drogas. Rev Bras Psiquiatr. 2000;22(2):32-6.

4. McKinnon SA, O'Rourke KM, Thompson SE, Berumen JH. Alcohol use and abuse by adolescents: the impact of living in a border community. J Adolesc Health. 2004;34(1):88-93. 
5. Silverman JG, Raj A, Mucci LA, Hathaway JE. Dating violence against adolescent girls and associated substance use, unhealthy weight control, sexual risk behavior, pregnancy, and suicidality. JAMA. 2001;286(5):572-9.

6. Brown SA, Tapert SF, Granholm E, Delis DC. Neurocognitive functioning of adolescents: effects of protracted alcohol use. Alcohol Clin Exp Res. 2000;24(2):164-71.

7. NHTSA. Traffic Safety Facts. 2010 Data; 2012. 8. Noto AR, Moreira FG. Prevenção ao uso indevido de drogas: conceitos básicos e sua aplicação na realidade brasileira. In: Silveira D, Moreira F, organizadores, editors. Panorama atual de drogas e dependências. São Paulo: Atheneu; 2006. p. 313-8.

9. Werch C, Moore M, DiClemente CC, Owen DM, Jobli E, Bledsoe R. A sport-based intervention for preventing alcohol use and promoting physical activity among adolescents. J Sch Health. 2003;73(10):380-8.

10. Van Rensburg KJ, Taylor A, Benattayallah A, Hodgson T. The effects of exercise on cigarette cravings and brain activation in response to smoking-related images. Psychopharmacology. 2012;221(4):659-66.

11. Lisha NE, Sussman S. Relationship of high school and college sports participation with alcohol, tobacco, and illicit drug use: A review. Addict Behav. 2010;35(5):399-407.

12. Fredricks JA, Eccles JS. Is extracurricular participation associated with beneficial outcomes? Concurrent and longitudinal relations. Dev Psychol. 2006;42(4):698-713.

13. Baumert PW, Jr., Henderson JM, Thompson NJ. Health risk behaviors of adolescent participants in organized sports. J Adolesc Health. 1998;22(6):460-5.

14. Papaioannou A, Karastogiannidou C, Theodorakis Y. Sport involvement, sport violence and health behaviours of Greek adolescents. Eur J Public Health. 2004;14(2):168-72.

15. Pate R, Heath G, Dowda M, Trost S. Association between physical activity and other health behaviors in a representative sample of US adolescents. Am J Public Health. 1996;86(11):1577-81.

16. Rainey CJ, McKeown RE, Sargent RC, Valois RF. Patterns of tobacco and alcohol use among sedentary, exercising, non-athletic, and athletic youth. J Sch Health 1996;66(1):27-32.

17. Ferron C, Narring F, Cauderay M, Michaud PA. Sport activity in adolescence: Associations with health perceptions and experimental behaviours. Health Educ Res. 1999;14(2):225-33.

18. Winnail S, Valois R, Dowda M, McKeown R, Saunders R, Pate R. Athletics and substance use among public high school students in a southern state. Am J Health Stud 1997;13:187-94.

19. Wichstrøm T, Wichstrøm L. Does sports participation during adolescence prevent later alcohol, tobacco and cannabis use? Addiction. 2009;104(1):138-49.

20. Page R, Hammermeister J, Scanlan A, Gilbert L. Is school sports participation a protective factor against adolescent health risk behaviors? J Health Educ 1998;29:186-92.

21. Naylor AH, Gardner D, Zaichkowsky L. Drug use patterns among high school athletes and nonathletes. Adolescence. 2001;36(144):627-39.

22. Bedendo A, Opaleye ES, Andrade AL, Noto AR. Heavy episodic drinking and soccer practice among high school students in Brazil: the contextual aspects of this relationship. BMC Public Health. 2013;13:247.

23. Garry JP, Morrissey SL. Team sports participation and risk-taking behaviors among a biracial middle school population. Clin J Sport Med. 2000;10(3):185-90.

24. Davis T, Arnold C, Nandy I, Bocchini J, Gottlieb A, George R, et al. Tobacco use among male high school athletes. J Adolesc Health 1997;21(2):97-101.

25. Jones RB, Moberg DP. Correlates of smokeless tobacco use in a male adolescent population. Am J Public Health. 1988;78(1):61-3.

26. van den Berg P, Neumark-Sztainer D, Cafri G, Wall M. Steroid use among adolescents: longitudinal findings from Project EAT. Pediatrics. 2007;119(3):476-86.

27. Mays D, DePadilla L, Thompson NJ, Kushner HI, Windle M. Sports participation and problem alcohol use: A multi-wave national sample of adolescents. Am J Preventive Med. 2010;38(5):491-8.

28. Peretti-Watel P, Beck F, Legleye S. Beyond the U-curve: the relationship between sport and alcohol, cigarette and cannabis use in adolescents. Addiction. 2002;97(6):707-16.

29. Moore MJ, Werch CEC. Sport and physical activity participation and substance use among adolescents. J Adolesc Health. 2005;36(6):486-93.

30. Kulig K, Brener ND, McManus T. Sexual activity and substance use among adolescents by category of physical activity plus team 
sports participation. Arch Pediatr Adolesc Med. 2003;157(9):905-12.

31. Peretti-Watel $P$, Guagliardo V, Verger $P$, Pruvost J, Mignon P, Obadia Y. Sporting activity and drug use: Alcohol, cigarette and cannabis use among elite student athletes. Addiction. 2003;98(9):1249-56.

32. Terry-McElrath YM, O'Malley PM, Johnston LD. Exercise and substance use among American youth, 1991-2009. Am J Prev Med. 2011;40(5):530-40.

33. Grossbard J, Hummer J, LaBrie J, Pederson E, Neighbors C. Is substance use a team sport? Attraction to team, perceived norms, and alcohol and marijuana use among male and female intercollegiate athletes. J Appl Sport Psychol. 2009;21(3):247-61.

34. Ryan RM, Deci EL. Self-determination theory and the facilitation of intrinsic motivation, social development, and well-being. Am Psychol. 2000;55(1):68-78.

35. Locatelli D, Sanchez Z, Opaleye E, Carlini C, Noto AR. Socioeconomic influences on alcohol use patterns among private school students in São Paulo. Rev Bras Psiquiatr. 2012;34(2):193-200.

36. Bronfenbrenner U. Ecological models of human development. 2nd ed. New York: Elsevier Science; 1994.

37. Hong JS, Huang H, Sabri B, Kim JS. Substance abuse among Asian American youth: An ecological review of the literature. Child Youth Serv Rev. 2011;33(5):669-77.

38. Hong JS, Lee NY, Grogan-Kaylorb A, Huang H. Alcohol and tobacco use among South Korean adolescents: An ecological review of the literature. Child Youth Serv Rev. 2011;33(7):1120-6.

39. Peck SC, Vida M, Eccles JS. Adolescent pathways to adulthood drinking: Sport activity involvement is not necessarily risky or protective. Addiction. 2008;103(Suppl 1):69-83.

40. Pinsky I, El Jundi SA. Alcohol advertising and alcohol consumption among youngsters: review of the international literature. Rev Bras Psiquiatr. 2008;30:362-74.

41. Abad JRR, Ruiz-Juan F, Rivera JIZ. Alcohol $\mathrm{y}$ tabaco en adolescentes españoles y mexicanos y su relación con la actividad físico-deportiva y la familia. Rev Panam Salud Pública. 2012;31(3):211-20.

42. Eccles JS, Barber BL. Student council, volunteering, basketball, or marching band: What kind of extracurricular involvement matters? J Aadolesc Res. 1999;14(1):10-43.
43. Barber B, Stone M, Hunt J, Eccles J. Benefits of activity participation: The role of identity affirmation and peer group norm sharing. In: Mahoney J, Larson, RW, Eccles, JS, organizadores, editor. Organized activities as contexts of development: Extracurricular activities, after-school and community programs. New Jersey: Erlbaum; 2005. p. 185-210.

44. Steinberg L. A Social Neuroscience Perspective on Adolescent Risk-Taking. Dev Rev. 2008;28(1):78-106.

45. Ruiz-Juan F, Cruz-Sánchez E, GarcíaMontes ME. Motivos para la práctica deportiva y su relación con el consumo de alcohol y tabaco en jóvenes españoles. Salud Publica Mex. 2009;51(6):496-504.

46. Kuntsche E, Knibbe R, Gmel G, Engels R. Why do young people drink? A review of drinking motives. Clin Psychol Rev. 2005;25(7):841-61.

47. Smoll F, Smith R. Psychology of the young athlete, stress-related maladies and remedial approaches. Sports Med. 1990;37(5):1021-46.

48. Frade IF, De Micheli D, Monezi Andrade AL, de Souza-Formigoni ML. Relationship between stress symptoms and drug use among secondary students. Spanish J Psychol. 2013;16:E4.

49. Belanger RE, Ohl F, Berchtold A, Akre C, Suris JC. Social contexts of sports-practicing youths' hazardous drinking. Swiss Med Wkly. 2012;142:w13526.

50. Lorente FO, Souville M, Griffet J, Grelot L. Participation in sports and alcohol consumption among French adolescents. Addict Behav. 2004;29(5):941-6. 